\section{Second malignancies after treatment of childhood non-Hodgkin lymphoma - a report of the Berlin-Frankfurt-Muenster study group}

\author{
Olga Moser, ${ }^{1}$ Martin Zimmermann, ${ }^{2}$ Ulrike Meyer, ${ }^{3}$ Wolfram Klapper, ${ }^{4}$ \\ Ilske Oschlies, ${ }^{4}$ Martin Schrappe, ${ }^{5}$ Andishe Attarbaschi, ${ }^{6}$ Georg Mann, ${ }^{6}$ \\ Felix Niggli, ${ }^{7}$ Claudia Spix, ${ }^{8}$ Udo Kontny, ${ }^{1}$ Thomas Klingebiel, $^{9}$ Alfred Reiter, ${ }^{3}$ \\ Birgit Burkhardt ${ }^{10}$ and Wilhelm Woessmann ${ }^{11}$ \\ ${ }^{1}$ Division of Pediatric Hematology and Oncology, RWTH-Aachen University, Aachen, \\ Germany; ${ }^{2}$ Department of Pediatric Hematology and Oncology, Medical School Hannover, \\ Hannover, Germany; ${ }^{3}$ Department of Pediatric Hematology and Oncology, Justus Liebig- \\ University Giessen, Giessen, Germany; ${ }^{4}$ Department of Pathology, Hematopathology \\ Section and Lymph Node Registry, University Hospital Schleswig Holstein, Campus Kiel, \\ Kiel, Germany; ${ }^{5}$ Department of Pediatric Hematology and Oncology, Children's University \\ Hospital, University Hospital Schleswig Holstein, Campus Kiel, Kiel, Germany; \\ ${ }^{6}$ Department of Pediatric Hematology and Oncology, St. Anna Children's Hospital, \\ Department of Pediatrics and Adolescent Medicine, Medical University of Vienna, Vienna, \\ Austria; ${ }^{7}$ Department of Pediatric Hematology and Oncology, Children's University Hospital \\ Zurich, Zurich, Switzerland; ${ }^{8}$ German Childhood Cancer Registry (GCCR) at Institute of \\ Medical Biostatistics, Epidemiology, and Informatics (IMBEI) of the Mainz University \\ Medical Center, Mainz, Germany; ${ }^{9}$ Department of Pediatric Hematology and Oncology, \\ Goethe University Frankfurt, Frankfurt, Germany; ${ }^{10}$ Pediatric Hematology and Oncology, \\ University Hospital Muenster, Muenster, Germany and ${ }^{11}$ Department of Pediatric \\ Hematology and Oncology, University Medical Center Hamburg-Eppendorf, Hamburg, \\ Germany
}

\section{ABSTRACT}

S econd malignant neoplasms (SMN) pose a concern for survivors of childhood cancer. We evaluated incidence, type and risk factors for SMN in patients included in Berlin-Frankfurt-Muenster protocols for childhood non-Hodgkin lymphoma.3,590 patients $<15$ years of age at diagnosis, registered between 01/1981 and 06/2010, were analyzed. SMN were reported by the treating institutions and the German Childhood Cancer Registry. After a median follow-up of 9.4 years (quartile [O] range, Q1 6.7 and $\mathrm{Q} 3$ 12.1) $95 \mathrm{SMN}$ were registered (26 carcinomas including nine basal cell carcinomas, 21 acute myeloid leukemias/myelodysplastic syndromes, 20 lymphoid malignancies, 12 central nervous system [CNS]-tumors, and 16 others). Cumulative incidence at 20 years was $5.7 \pm 0.7 \%$, standard incidence ratio, excluding basal cell carcinomas, was 19.8 (95\% Confidence Interval [CI]: 14.5-26.5). Median time from initial diagnosis to second malignancy was 8.7 years (range, 0.2-30.3 years). Acute-lymphoblasticleukemia-type therapy, cumulative anthracycline dose, and cranial radiotherapy for brain tumor-development were significant risk factors in univariate analysis only. In multivariate analysis including risk factors significant in univariate analysis, female sex (hazard ratio [HR] 1.87, 95\% CI: 1.23-2.86, $P=0.004$ ), CNS-involvement (HR 2.24, 95\% CI: 1.03-4.88, $P=0.042$ ), lymphoblastic lymphoma (HR 2.60, 95\% CI: 1.69-3.97, $P<0.001$ ), and cancer-predisposing condition (HR 11.2, 95\% CI: 5.52-22.75, $P<0.001$ ) retained an independent risk. Carcinomas were the most frequent SMN after non-Hodgkin lymphoma in childhood followed by acute myeloid leukemia and lymphoid malignancies. Female sex, lymphoblastic lymphoma, CNS-involvement, or/and known cancer-predisposing condition were risk factors for $\mathrm{SMN}$-development. Our findings set the basis for individualized long-term follow-up and risk assessment of new therapies. 


\section{Introduction}

Second malignant neoplasms (SMN) represent a serious long-term risk after treatment of children with cancer. Reported cumulative incidences of SMN after childhood cancer vary between 3 and $11 \%$ at $20-30$ years depending on the first cancer, type of treatment and cumulative drug doses. ${ }^{1.5}$ No lifetime cumulative incidence has been established so far. A recently published study of the German Childhood Cancer Registry (GCCR) has shown a cumulative incidence of $8.3 \%$ within 35 years after first cancer in childhood. ${ }^{6}$ Analysis of SMN after treatment for childhood non-Hodgkin lymphoma (NHL) showed cumulative incidences between 3 and $10 \%$ at $20-30$ years after NHL diagnosis; the reported standard incidence ratios (SIR) for SMN varied between 2.4 and 12.0. ${ }^{1-5,6-10}$ Most information is derived from cancer registries in population-based studies on SMN after childhood cancer. ${ }^{1-9}$ These studies lack detailed information about the NHL-subtypes and specific treatments. Two previous studies focused on SMN after childhood NHL.,10 One of them limited the analysis to 5-year survivors of NHL, thus omitting most of the hematologic SMN that occur within 5 years of first diagnosis. ${ }^{9}$ Two further studies about late health outcomes after treatment of childhood NHL included incidences of SMN, however, restricted the analysis to 5- and 10-year survivors of NHL, respectively. ${ }^{11,12}$

Studies on SMN after NHL in adulthood report an increased incidence of leukemia and solid tumors. ${ }^{13,14} \mathrm{~A}$ meta-analysis concerning the risk of SMN in adult NHL survivors detected a 1.88-fold increased risk for SMN in comparison with the general population. ${ }^{14}$

Treatment strategies for children with NHL differ from those used in adults with respect to the cumulative drug doses and the application of radiation. Whereas radiotherapy was largely omitted during the 1970s in the pediatric Berlin-Frankfurt-Muenster (BFM)-trials, it is nowadays applied in the treatment of most adults with NHL and bulky disease (Online Supplementary Tables S1 and S2).

In addition, the incidence of SMN may vary according to NHL subtype and treatment regimen, and may also be influenced by genetic and specific host factors.

We analyzed the incidence and type of SMN as well as risk factors for the development of SMN in a populationbased large cohort of children treated for NHL with uniform subtype-specific therapy regimens according to the NHL-BFM trials. Cumulative drug doses and the backbone of the NHL-BFM trials remained stable over the analysis period of 30 years.

\section{Methods}

From 01/1981 to 06/2010, 4,184 children from 99 BFM-institutions diagnosed with NHL were registered in the studies ALL/NHL-BFM-81, -83, -86, NHL-BFM-90, -95, ALCL-99, EUROLB-02, LBL-Register and B-NHL-BFM-04 after informed consent of the patient and/or guardians. The studies were conducted according to the Declaration of Helsinki. Approval was obtained from ethical committees of the investigators. 3,590 patients $<15$ years of age at diagnosis, were included in the analysis by $04 / 2017$ (Figure 1).

NHL were classified using classifications at the time of diagno- sis $^{15,16,17}$ (see the Online Supplementary Appendix). Central reference pathology- and/or immunology-review was performed in over $90 \%$ of cases.

Patients were stratified according to the St Jude staging system ${ }^{18}$ and treated on one of the NHL-BFM-protocols listed above. Patients with lymphoblastic lymphoma (LBL) received an acutelymphoblastic-leukemia (ALL)-type BFM-regimen consisting of eight drug inductions, consolidations, re-intensifications, and maintenance up to 2 years of therapy. Patients with mature B-cellNHL or anaplastic large-cell-lymphoma (ALCL) received two to six courses of 5-day block-type chemotherapy as previously reported. ${ }^{19-23}$ All cumulative drug- and radiation doses are listed in the Online Supplementary Tables S1 and S2.

Long-term follow-up of patients was assured by the NHL-BFM datacenter and the GCCR (general information on data collection by the GCCR: www.kinderkrebsregister.de/english/). Details on follow-up are described in the Online Supplementary Appendix.

For the purpose of this evaluation, histopathology information about the first and second malignancy was re-reviewed in all patients. If required, complementary affirmative investigations were performed by the central reference pathology department. Non-lymphoid and lymphoid malignancies fulfilling the criteria depicted below were considered as proven SMN. In cases of multiple SMN after NHL, only the first second cancer was counted.

\section{Statistical analysis}

The risk of SMN was estimated by cumulative incidence functions for SMN and death as competing events. Functions were compared using Gray test and $95 \%$ Confidence Intervals (CI) were calculated using standard methods. ${ }^{24}$

Survival after SMN was calculated from the date of SMN-diagnosis to the date of death or last follow-up, respectively. Probability of survival was calculated according to Kaplan-Meier. ${ }^{25}$

SIR were calculated as the ratio of the observed SMN and the expected number of malignancies, as described by Scholz-Kreisel et al. ${ }^{6}$ Age-, sex-, and calendar year-specific rates from the GCCR were used to calculate the expected numbers for cases below 15 years. These data are complete and cover all diagnoses reported here. Population-based incidence rates for cases older than 15 years were not available for the entire period and all diagnoses. Therefore, the SIR was calculated for a subgroup of the cohort without basal cell carcinoma.

The contribution of factors to the development of SMN was estimated with the Cox proportional-hazards model. The clinical and biologic features analyzed included age at first diagnosis, sex, NHL-entity, -stage, therapy-type and known cancer-predisposing condition (CPC). The doses (as per protocol) of epipodophyllotoxins, cyclophosphamide/ifosfamide (dose equivalence ratio 1:4), anthracyclines (dose equivalence ratio 1:1), cytarabine, asparaginase were analyzed, as well as stem-cell transplantation, and cranial radiotherapy. Statistical analysis was performed using SAS program (SAS, version 9.4; SAS Institute Inc, Cary, NC, USA).

\section{Results}

\section{Patient characteristics}

Patient characteristics are shown in Table 1. Because of the age restriction applied by the GCCR until 2008, we included only patients $<15$ years at first diagnosis of $\mathrm{NHL}$ in our analysis to ensure the most accurate assessment of the incidence of SMN in the study population. The analyzed group included 57 patients with known CPC, namely primary and secondary immune deficiencies, Gorlin-, mismatch-repair-deficiency syndromes, neurofibro- 
matoses, Fanconi anemia. There was no systematic screening for CPC in the study population. Reported patients were identified by the presence of clinical immune deficiency, and/or signs of the syndromes stated above. Investigations for CPC were prompted by multiple-cancer development and/or diminished therapy toler- ance in some cases. Six of 57 patients with CPC received radiotherapy. Altogether, $24.7 \%$ of all 3,590 patients received ALL-type therapy and 75.3\% B-NHL-type regime. 450 patients in the analyzed cohort $(12.5 \%)$ suffered from relapse of the NHL. Treatment for relapsed disease consisted of individualized chemotherapy. Two hun-

Table 1. Analysis of risk factors for the development of second malignant neoplasms after non-Hodgkin lymphoma in children $<15$ years of age at diagnosis, treated in one of the consecutive NHL-BFM studies NHL-BFM-81, through EURO-LB-02/B-NHL-04.

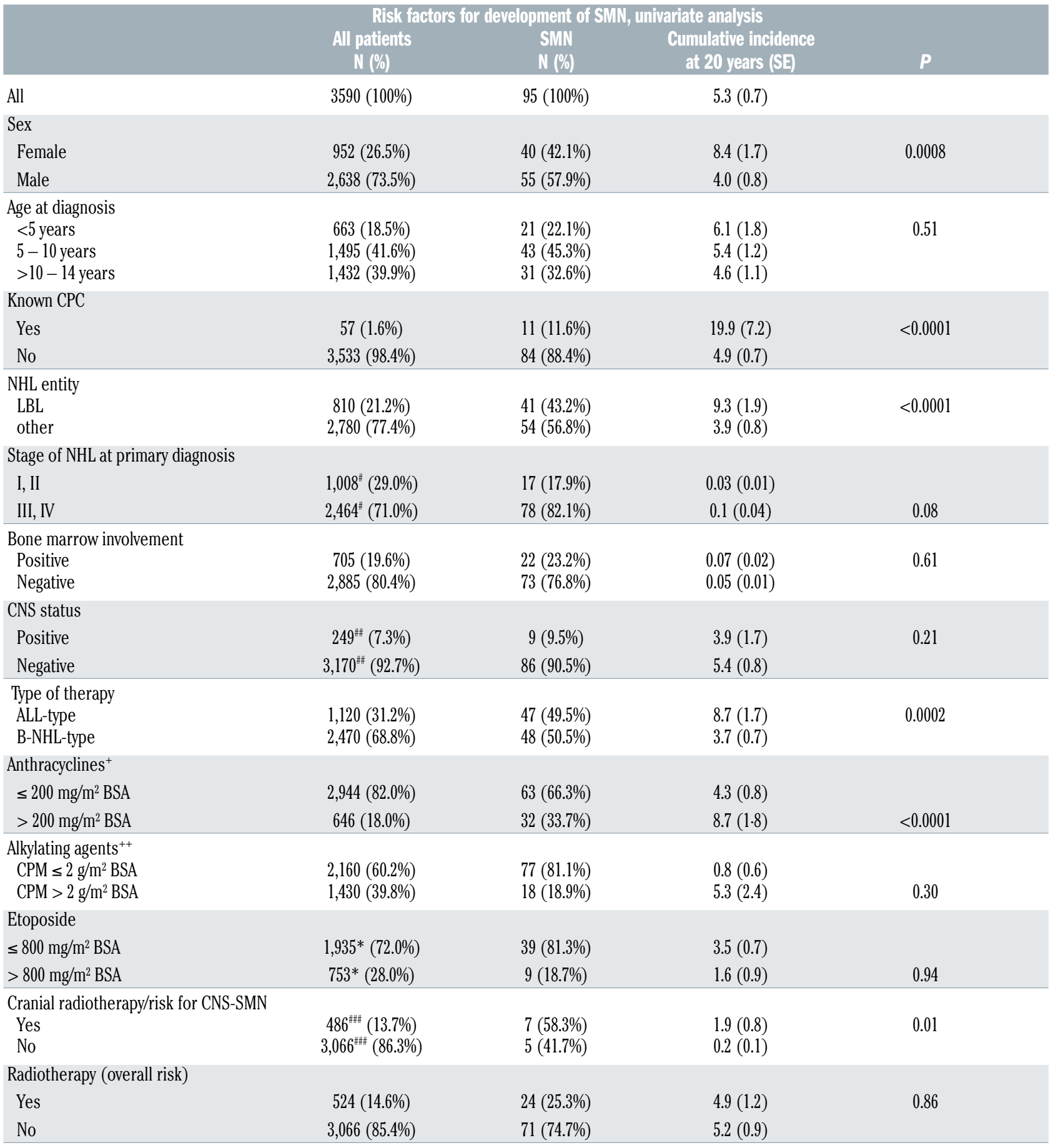

Cumulative drug doses were calculated as per protocol. ${ }^{+}$Doxorubicin equivalent dose was calculated at 1:1 ratio for the used anthracyclines (doxorubicin and daunorubicin). "Alkylating agents: conversion factor for cyclophosphamide equivalent dose was 1:4 (i.e., $1 \mathrm{mg}$ cyclophosphamide equals $4 \mathrm{mg}$ ifosfamide); *indicates only patients receiving B-NHL-type therapy; \# information about stage of disease missing in 118 patients; "\#information about CNS involvement missing in 171 patients; ; information about cranial radiotherapy missing in 38 patients. SMN: second malignant neoplasm; NHL: non-Hodgkin lymphoma; ALCL: anaplastic large-cell lymphoma; ALL: acute lymphoblastic leukemia; BNHL: mature B-cell lymphoma; BSA: body surface area; CI: Confidence Interval; CNS: central nervous system; CPC: cancer-predisposing condition, CPM: cyclophosphamide; HR: hazard ratio; LBL: lymphoblastic lymphoma; nfc: not further classified; NHL: non-Hodgkin Lymphoma; N: number; SE: standard error; SMN: second malignant neoplasm. 
dred and fifty-two patients received consolidation by stem cell transplantation. Patients developing any kind of SMN did not significantly differ from those who did not with respect to the age at diagnosis, stage of NHL, or bone-marrow involvement.

With a median follow-up of 9.4 years (quartile [Q] range, Q1 6.7 and $\mathrm{Q} 3$ 12.1), the probability of survival at 20 years was $83 \%$, standard error $(\mathrm{SE})=0.01$ for the total group of 3,590 patients.

\section{Second malignant neoplasms}

Ninety-five SMN were documented: 26 carcinomas (including nine basal-cell carcinomas), 21 acute myeloid leukemia (AML)/myelodysplastic syndrome (MDS), 20 second lymphoid malignancies (12 NHL, four ALL, four Hodgkin disease), 12 malignant CNS-tumors, five sarcomas, five malignant melanomas, and seven other SMN (Figure 1, Table 3).

Second lymphoid malignancy was considered to be proven when (i) in lymphoid malignancy there was a lineage change, i.e., $\mathrm{B}$ lineage to $\mathrm{T}$ lineage and vice versa, (ii) in lymphoid malignancy there was a change with respect to the differentiation compartment, i.e., from a precursor $\mathrm{B}$ (or T) neoplasm to mature B (or T) neoplasm or vice versa, (iii) in Burkitt lymphoma/Burkitt leukemia there was a MYC rearrangement at different break points between the first and the second cancer or (iiii) in mature B-NHL there was a different immunoglobulin light-chain restriction between the first and second malignancy i.e., $\lambda$ to $\kappa$ or vice versa. Not included as SMN were eight laterecurring lymphoid malignancies with different clonal rearrangements of immunoglobulin $\mathrm{H}$ - and T-cell-receptor-genes, but no differences with respect to discriminators listed above (i-iiii), since clonal evolution in these cases could not be excluded. Evaluation of clonal origin via multiplex-polymerase chain reaction (BIOMED-2 method $^{26}$ ) was performed for all available tumor samples in cases of second lymphoid malignancy.

We also observed 20 benign neoplasms (eight low-grade meningeomas, five adenomas, four osteochondromas, one pilomatrixoma, chondroblastoma, neurinoma each).

The SIR for all SMN without basal cell carcinoma was 19.8 (95\% CI: 14.5-26.5).

Cumulative incidence of all SMN at 20 years was $5.3 \%$ $(\mathrm{SE}=0.7)$ (Figure 2A). Cumulative incidences of distinct SMN-groups are depicted in Figure 3 . The median age at diagnosis of NHL within the population of $<15$ years was

Table 2. Stepwise Cox regression analysis of risk factors for the development of second malignant neoplasms after non-Hodgkin lymphoma in children $<15$ years of age at diagnosis, treated according to one of the consecutive NHL-BFM studies NHL-BFM-81, through EURO-LB02/B-NHL-04.

\begin{tabular}{lcc} 
Sex & NR $(95 \%$ Cl) & $P$ \\
$\quad$ female $v s$. male & $1.87(1.23-2.86)$ & 0.004 \\
$\begin{array}{l}\text { NHL entity } \\
\text { LBL } v \text { s. other } \\
\text { CNS involvement } \\
\text { positive } v \text { s. negative }\end{array}$ & $2.6(1.69-3.97)$ & $<0.001$ \\
\hline
\end{tabular}

\section{Known CPC}

yes $v s$. no

CI: Confidence Interval; CNS: central nervous system; CPC: cancer-predisposing condition; HR: hazard ratio; LBL: lymphoblastic lymphoma; NHL: non-Hodgkin lymphoma.

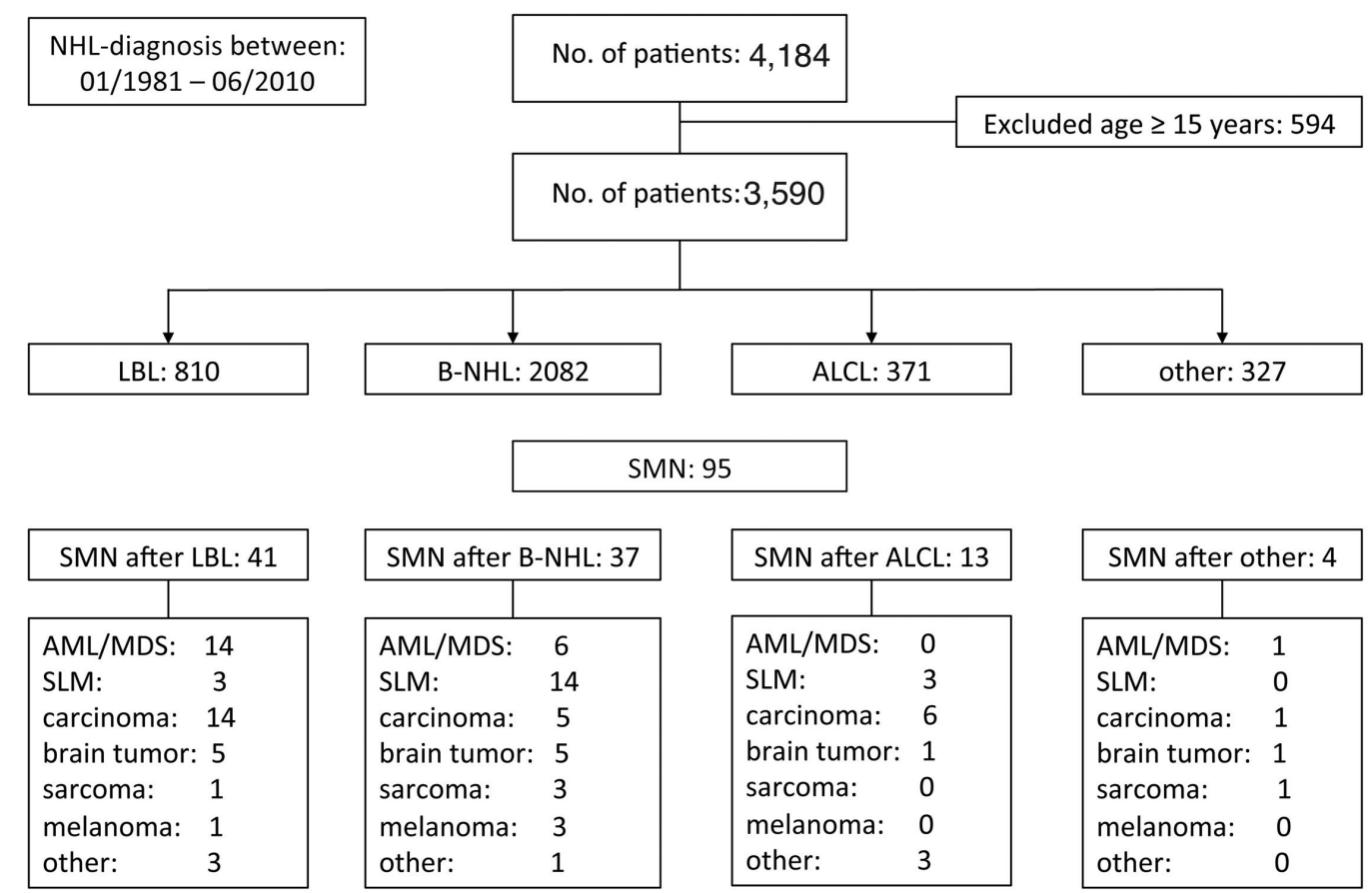

Figure 1. Consort diagram: numbers of analyzed patients and second malignant neoplasms. ALCL: anaplastic large-cell lymphoma; AML/MDS: acute myeloid leukemia/myelodysplastic syndrome; LBL: Iymphoblastic lymphoma; NHL: non-Hodgkin lymphoma, No.: number; SLM: second lymphoid malignancy, SMN: second malignant neoplasm. 
A

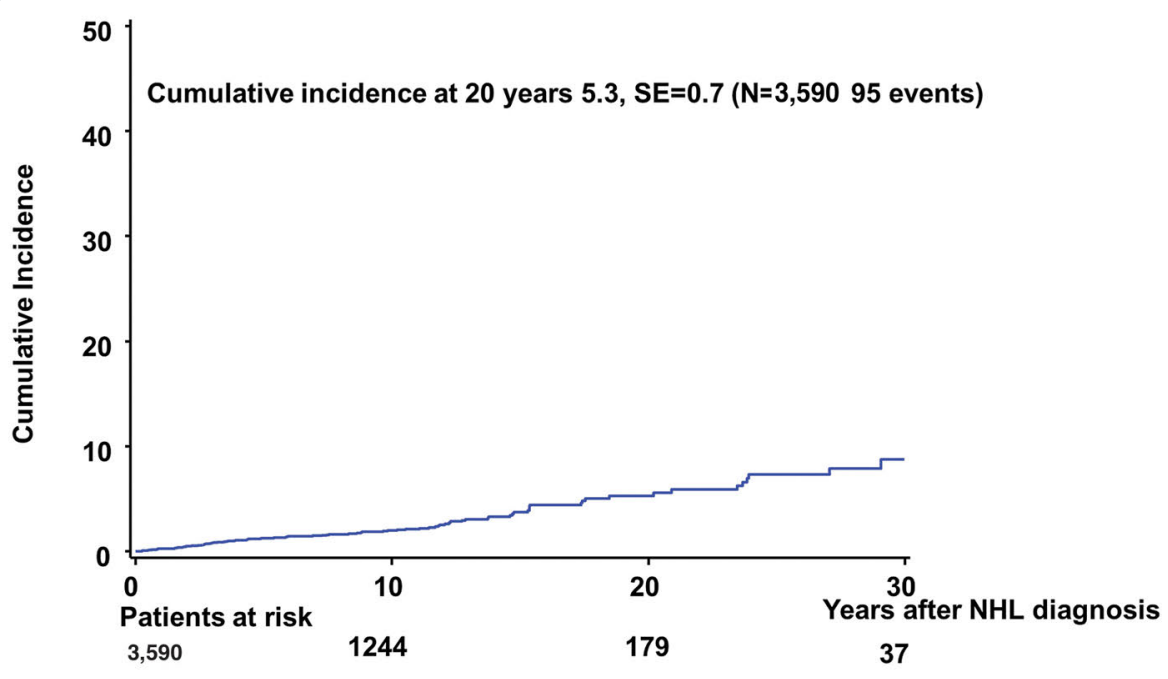

B

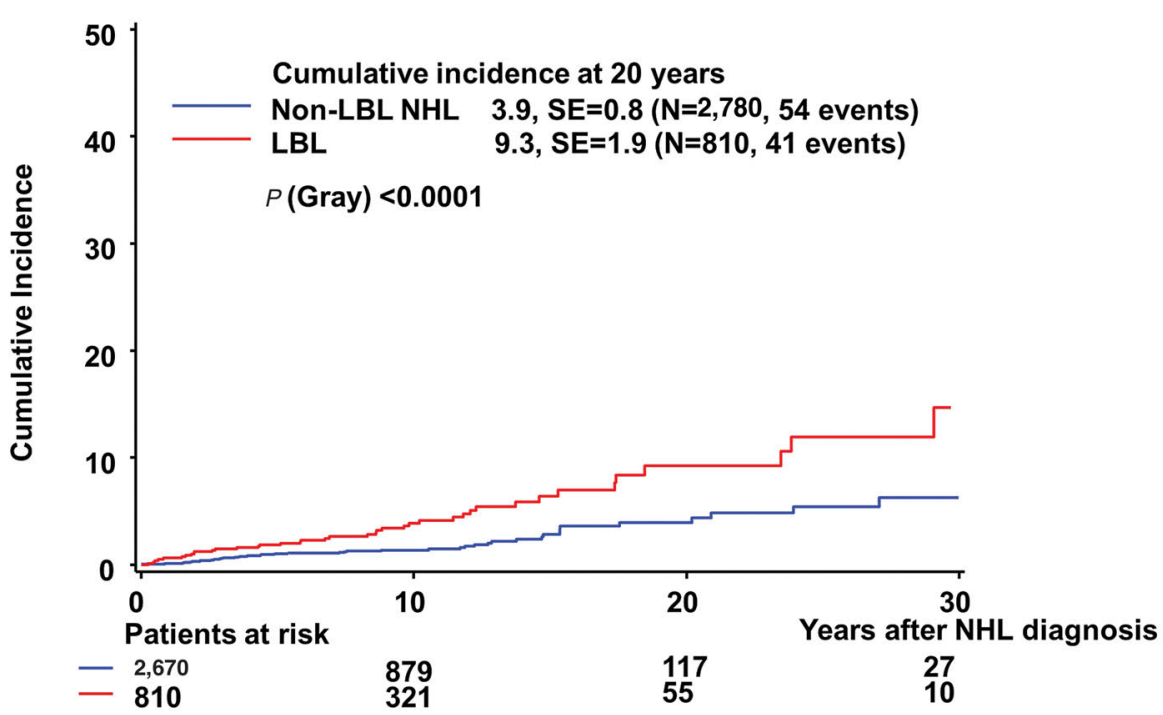

C

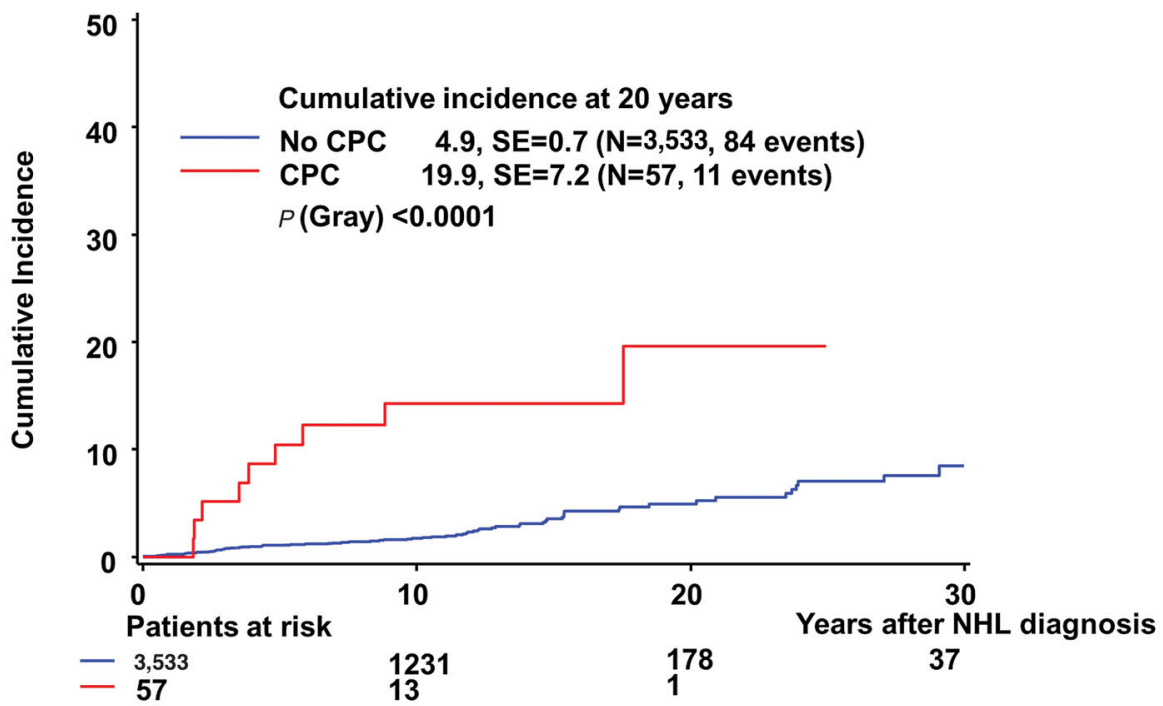

Figure 2. Estimated cumulative incidence of second malignant neoplasms in patients with non-Hodgkin lymphoma $(\mathrm{NHL})<15$ years of age at diagnosis, treated in one of the consecutive NHL-BFM studies NHL-BFM81, through EURO-LB-02/B-NHL-04. (A) Cumulative incidence of second malignant neoplasms (SMN) for all analyzed patients $(5.3 \% \pm 0.7)$. (B) Cumulative incidence of SMN for patients with lymphoblastic lym phoma (LBL) (9.3 \pm 1.9$)$ compared to patients with other NHL (Non-LBL $\mathrm{NHL})(3.9 \pm 0.8)$. (C) Cumulative incidence of SMN for NHL-patients with known cancer-predisposing condition (CPC) $(19.9 \pm 7.2)$ compared to patients without known CPC $(4.9 \pm 0.7)$. SE: standard error. 
8.1 years (range, 0.3-14.9 years) for patients who subsequently developed a SMN. The median time from first diagnosis of NHL to SMN was 8.7 years (range, 0.2-30.3 years). The median time to development of all carcinomas was 15.9 years (range, 0.7-30.3 years), and 11.6 years (range, 0.7-23.7 years) excluding basal-cell carcinomas. The median time was 3.1 years (range, 0.3-8.7 years) for AML/MDS, 5.7 years (range, 1.5-20.2 years) for second lymphoid malignancy, and 8.6 years (range, 0.8-17.3 years) for CNS-tumors, respectively (Table 3). Carcinomas of the gastrointestinal tract and basal-cell carcinoma were the most frequent carcinomas (nine cases each) (Table 3). Six of the nine gastrointestinal tract-carcinomas occurred after a T-cell LBL.

\section{Univariate analysis of risk factors (Table 1)}

Cumulative incidence of SMN was significantly higher among patients with $\operatorname{LBL}(9.3 \%$ at 20 years, $\mathrm{SE}=1.9 \%)$

Table 3. Characteristics and outcome of patients with second malignant neoplasms after non-Hodgkin lymphoma in children.

\begin{tabular}{|c|c|c|c|c|c|c|c|c|c|}
\hline \multirow[b]{2}{*}{$\begin{array}{l}\text { No. } \\
\text { of pts }\end{array}$} & \multirow[b]{2}{*}{$\begin{array}{l}\operatorname{Sex} \\
M / F\end{array}$} & \multicolumn{3}{|c|}{ Second malignant neoplasm } & \multicolumn{5}{|c|}{ Primary NHL } \\
\hline & & $\begin{array}{c}\text { SMN type } \\
\text { [no. of patients] }\end{array}$ & $\begin{array}{l}\text { Latency } \\
\text { years } \\
\text { medlan } \\
\text { (range) }\end{array}$ & $\begin{array}{l}\text { Outcome: } \\
\text { alive/death/3rd } \\
\text { malignancy/LFU }\end{array}$ & $\begin{array}{l}\text { Type } \\
\text { of NHL } \\
\text { [no. of } \\
\text { patients] }\end{array}$ & $\begin{array}{c}\text { Age } \\
\text { at Dx years } \\
\text { median } \\
\text { (range) }\end{array}$ & $\begin{array}{l}\text { Stage } \\
\text { of disease } \\
\text { I/II/III/IV }\end{array}$ & $\begin{array}{c}\text { Therapy } \\
\text { type } \\
\text { ALL/B-NHL }\end{array}$ & $\begin{array}{l}\text { Radio-therapy } \\
\text { Yes/no/ } \\
\text { unknown }\end{array}$ \\
\hline 21 & $12 / 9$ & $\begin{array}{c}\text { MDS and AML } \\
\text { MDS-AML del(5),del(7) } \\
\text { and/or complex karyotype [6] } \\
\text { AML t(11q23) [4] } \\
\text { AML normal karyotype [5] } \\
\text { AML other [3] } \\
\text { AML no cytogenetics [3] }\end{array}$ & $\begin{array}{c}3.1 \\
(0.3-8.7)\end{array}$ & $5 / 14 / 1 / 2$ & $\begin{array}{l}\text { T-LBL [9] } \\
\text { pB-LBL [5] } \\
\text { BL/B-AL [5] } \\
\text { B-NHL [1] } \\
\text { NHL nfc [1] }\end{array}$ & $\begin{array}{c}3.4 \\
(0.7-14.6)\end{array}$ & $1 / 0 / 8 / 12$ & $15 / 6$ & $4 / 15 / 2$ \\
\hline 20 & $13 / 7$ & $\begin{array}{c}\text { Second lymphoid malignancy } \\
\text { T-LBL [3] } \\
\text { ALL [4] } \\
\text { BL/B-AL [3] } \\
\text { B-NHL [3] } \\
\text { ALCL [1] } \\
\text { PTCL [2] } \\
\text { HD [4] }\end{array}$ & $\begin{array}{c}5.7 \\
(1.5-20.2)\end{array}$ & $7 / 7 / 5 / 2$ & $\begin{array}{l}\text { T-LBL [3] } \\
\text { BL/B-AL [4] } \\
\text { B-NHL [10] } \\
\text { ALCL [3] }\end{array}$ & $\begin{array}{c}8.1 \\
(0.7-14.9)\end{array}$ & $2 / 3 / 13 / 2$ & $3 / 17$ & $1 / 19 / 0$ \\
\hline 12 & $9 / 3$ & $\begin{array}{l}\text { CNS malignant tumors } \\
\text { Glioblastoma multiforme [3] } \\
\text { Anapl. Astrocytoma }{ }^{\circ} \text { III [3] } \\
\text { Anapl. Meningeoma }{ }^{\circ} \text { III [3] } \\
\text { Medulloblastoma [1] } \\
\text { undifferentiated [2] }\end{array}$ & $\begin{array}{c}8.6 \\
(0.8-17.3)\end{array}$ & $3 / 6 / 1 / 3$ & $\begin{array}{c}\text { T-LBL [4] } \\
\text { pB-LBL [1] } \\
\text { BL/B-AL [2] } \\
\text { B-NHL [3] } \\
\text { ALCL [1] } \\
\text { PTCL [1] }\end{array}$ & $\begin{array}{c}6.8 \\
(1.9-11.3)\end{array}$ & $0 / 3 / 6 / 3$ & $6 / 6$ & $7 / 2 / 3$ \\
\hline 26 & $13 / 13$ & $\begin{array}{c}\text { Carcinoma } \\
\text { Basal cell carcinoma [9] } \\
\text { Gastrointestinal tract [9] } \\
\text { Thyroid [4] } \\
\text { Breast [1] } \\
\text { NPC [1] } \\
\text { Renal [1] } \\
\text { Urothel [1] }\end{array}$ & $\begin{array}{c}15.9 \\
(0.7-30.3)\end{array}$ & $15 / 4 / 1 / 6$ & $\begin{array}{c}\text { T-LBL [13] } \\
\text { pB-LBL [1] } \\
\text { BL/B-AL [4] } \\
\text { B-NHL [1] } \\
\text { ALCL [6] } \\
\text { NHL n.f.c. [1] }\end{array}$ & $\begin{array}{c}9.5 \\
(1.6-13.3)\end{array}$ & $3 / 1 / 18 / 4$ & $15 / 11$ & $8 / 14 / 4$ \\
\hline 5 & $4 / 1$ & $\begin{array}{l}\frac{\text { Sarcoma }}{\text { RMA [1] }} \\
\text { Leiomyosarcoma [1] } \\
\text { Clear cell sarcoma [1] } \\
\text { Ewing sarcoma [1] } \\
\text { Osteosarcoma [1] }\end{array}$ & $\begin{array}{c}8.6 \\
(1.9-15.3)\end{array}$ & $3 / 2 / 0 / 0$ & $\begin{array}{c}\text { T-LBL [1] } \\
\text { BL/B-AL [2] } \\
\text { B-NHL [1] } \\
\text { NHL nfc[1] }\end{array}$ & $\begin{array}{c}5.5 \\
(1.3-10.4)\end{array}$ & $0 / 2 / 1 / 2$ & $1 / 4$ & $1 / 4 / 0$ \\
\hline 4 & $2 / 2$ & Malignant Melanoma & $\begin{array}{c}12.3 \\
(1.6-30.0)\end{array}$ & $3 / 0 / 0 / 1$ & $\begin{array}{l}\text { T-LBL [1] } \\
\text { BL [1] } \\
\text { B-NHL [2] }\end{array}$ & $\begin{array}{c}10.5 \\
(5.3-13.7)\end{array}$ & $0 / 1 / 2 / 1$ & $1 / 3$ & $1 / 3 / 0$ \\
\hline 7 & $2 / 5$ & $\begin{array}{c}\text { Other } \\
\text { Extrarenal rhabdoid tumor [1] } \\
\text { Seminoma [1] } \\
\text { Malignant Phylloides [1] } \\
\text { Desmoid-Fibromatosis [1] } \\
\text { Hemangioendothelioma [1] } \\
\text { LCH [1] } \\
\text { Polycythemia rubra vera [1] }\end{array}$ & $\begin{array}{c}6.3 \\
(0.2-14.7)\end{array}$ & $2 / 3 / 1 / 2$ & $\begin{array}{l}\text { T-LBL [3] } \\
\text { B-NHL [1] } \\
\text { ALCL [3] }\end{array}$ & $\begin{array}{c}10.1 \\
(0.3-13.5)\end{array}$ & $0 / 1 / 5 / 1$ & $2 / 5$ & $0 / 7 / 0$ \\
\hline
\end{tabular}

ALCL: anaplastic large-cell lymphoma; B-NHL: mature B-cell lymphoma (other than BL/B-AL); BL/B-AL: Burkitt lymphoma /leukemia; HD: Hodgkin disease; LCH: Langenhans cell histiocytosis; LBL: lymphoblastic lymphoma; nfc: not further classified; NPC: nasopharyngeal carcinoma; PTCL: peripheral T-cell lymphoma; RMA: alveolary rhabdomyosarcoma. pts: points; no.: number; Dx: diagnosis. 
Table 4. Second malignant neoplasms after non-Hodgkin lymphoma in children in patients with known cancer predisposition condition.

\begin{tabular}{|c|c|c|c|c|c|c|c|c|c|c|}
\hline & $\begin{array}{l}\text { (years) } \\
\text { NHL-Dx }\end{array}$ & $\begin{array}{l}\text { Predisposing } \\
\text { condition }\end{array}$ & 1. NHL & $\begin{array}{l}\text { Therapy } \\
\text { type }\end{array}$ & $\mathrm{RT}^{*}$ & SMN & $\begin{array}{l}\text { Latency } \\
\text { time to } \\
\text { SMN } \\
\text { (years) }\end{array}$ & \multicolumn{3}{|c|}{$\begin{array}{l}\text { 3. malignant 4, malignant Outcome } \\
\text { neoplasm neoplasm }\end{array}$} \\
\hline M & 1.9 & Fanconi Anemia & BL & B-NHL & No & Medulloblastoma & 2.1 & MDS-RAEB/AML & - & Death of SMN \\
\hline M & 3.4 & Neurofibromatosis 1 & T-LBL & ALL-type & No & AML & 1.8 & - & - & Death of SMN \\
\hline M & 9.3 & ID n.f.c. & ALCL & B-NHL & No & DLBCL & 4.8 & Osteosarcoma & T-ALL & Death of SMN \\
\hline M & 9.6 & ID (organ transplant) & DLBCL & B-NHL & No & C-ALL & 4.4 & - & - & Death of SMN \\
\hline $\mathrm{F}$ & 1.3 & ID (organ transplant) & NHL n.f.c. & B-NHL & No & Leiomyosarcoma & 1.9 & - & - & alive \\
\hline $\mathrm{F}$ & 9.5 & NBS & PB & B-NHL & No & BL & 3.4 & T-ALL & - & Death of SMN \\
\hline $\mathrm{F}$ & 5.6 & NBS & DLBCL & B-NHL & No & ALCL & 3.8 & - & - & Death (other) \\
\hline $\mathrm{F}$ & 9.6 & $\begin{array}{c}\text { CMMRD } \\
\text { (compound heterozygote } \\
\text { PMS2-mutation) }\end{array}$ & DLBCL & B-NHL & No & c-ALL & 2.4 & Colon-CA & - & alive \\
\hline $\mathrm{F}$ & 1.2 & $\begin{array}{l}\text { CMMRD (mutation in } \\
\text { the } M S H 2 \text { gene) }{ }^{* *}\end{array}$ & T-LBL & ALL-type & No & B-NHL & 11.1 & $\begin{array}{l}\text { Phylloides- } \\
\text { tumor; }\end{array}$ & $\begin{array}{l}\text { Anaplastic } \\
\text { astrocytoma }\end{array}$ & alive \\
\hline $\mathrm{F}$ & 6.5 & $\begin{array}{l}\text { CMMRD (mutation } \\
\text { in } P M S 2 \text { gene) }\end{array}$ & T-LBL & ALL-type & No & Colon-CA & 9.8 & Urothel-CA & - & Death of SMN \\
\hline $\mathrm{F}$ & 6.9 & Gorlin-Goltz syndrome & T-LBL & ALL-type & Yes & NPC & 8.8 & - & $(\mathrm{t}$ & $\begin{array}{l}\text { Death } \\
\text { therapy related) }\end{array}$ \\
\hline
\end{tabular}

${ }^{*}$ Six of the 57 patients with known cancer predisposition condition in the study cohort received radiotherapy; * ${ }^{*}$ CMMRD diagnosis was first made after the occurrence of SMN. ALCL: anaplastic large-cell lymphoma; BL: Burkitt lymphoma; B-NHL: mature B-cell lymphoma (other than BL/B-AL); CA: carcinoma; DLBCL: diffuse large B-cell lymphoma; CMMRD: constitutive mismatch repair deficiency syndrome, caused by mutations in DNA mismatch repair genes PMS2, MSH6, MSH2 or MLH1; CPC: cancer predisposing condition; Dx: diagnosis; F: female; ID: immune deficiency; M: male; NBS: Nijmegen breakage syndrome; n.f.c.: not further classified; NHL: non-Hodgkin lymphoma; NPC: nasopharyngeal carcinoma; PB: plasmoblastic lymphoma; RT: radiotherapy; T-LBL: T-cell lymphoblastic lymphoma.

compared to patients with other NHL-entities $(3.9 \%$ at 20 years, $\mathrm{SE}=0.8 \%, P<0.0001$ ) (Figure $2 \mathrm{~B}$ ). In patients with LBL, both carcinomas and AML/MDS were the most common SMN (14 cases each), accounting for $72 \%$ of all SMN after LBL. The cumulative incidence of AML/MDS was $1.8 \%, \mathrm{SE}=0.5 \%$ after $\mathrm{LBL}$ compared to $0.1 \%, \mathrm{SE}=0.1 \%$ after mature B-NHL $(P<0.0001)$. Carcinomas had a higher cumulative incidence in LBL compared to mature B-NHL as well $(4.6 \%, \mathrm{SE}=1.5 \%$ vs. $2.5 \%, \mathrm{SE}=0.7 \%, P=0.04)$. Second lymphoid malignancy represented the largest SMN-group (34\%) after mature B-NHL. Female sex was a risk factor for SMN (20-year cumulative incidence 8.4\% vs. $4.0 \%$ in males, $P=0.0008$ ) (Table 1 ). Patients with known cancer predisposing condition (CPC) were at significantly higher risk for development of SMN (cumulative incidence $19.9 \%$ vs. $4.9 \%, P<0.0001$ ) (Figure $2 \mathrm{C}$ ). The majority of patients with CPC were female $(66.7 \%)$. The most frequent SMN in patients with CPC was another lymphoid malignancy (Table 3; Online Supplementary Table S3). With a median age of 6.4 years (range, 1.3-9.6 years) at primary NHL diagnosis, patients with CPC were younger than other patients with SMN. Neither disease stage nor bone marrow involvement at primary diagnosis were significant risk factors (Table 1).

Only patients with LBL received ALL-type therapy. Only ALL-type therapy contains $>200 \mathrm{mg} / \mathrm{m}^{2}$ doxorubicin equivalent. Accordingly, the cumulative incidence of SMN at 20 years was significantly higher after ALL-type therapy $(8.7 \%, \mathrm{SE}=1.7)$ compared to B-NHL-type therapy (3.7\%, SE=0.7; $P=0.0002)$ and among patients who had received $>200 \mathrm{mg} / \mathrm{m}^{2}$ doxorubicin equivalent (8.7, $\mathrm{SE}=1.8$ ) compared to patients receiving less (4.3, $\mathrm{SE}=0.8$, $P<0.0001)$. Exposure to etoposide was not a risk factor for AML/MDS (results not shown). $76 \%$ of patients who developed AML/MDS did not receive any epipodophyllotoxins. The use of high cumulative doses of alkylating agents was not associated with higher incidence of SMN (Table 1).

Patients who received cranial radiotherapy were at significantly higher risk for developing a second CNS malignancy $(P=0.01)$, (Table 1$)$.

Stem cell transplantation correlated with SMN-development among patients who received B-NHL-type therapy (cumulative incidence $10.4 \%, \mathrm{SE}=3.9$ vs. 2.8, $\mathrm{SE}=0.6$; $P=0.005)$.

Because the previous reports of SMN after childhood cancer did not include non-melanoma skin cancer, for comparison purpose we performed cumulative incidence analyses excluding basal-cell carcinoma. Cumulative incidence of all SMN excluding basal-cell carcinoma at 20 years was $4.8 \%$ ( $\mathrm{SE}=0.7$ ). Cumulative incidence of SMN remained significantly higher among patients with $\mathrm{LBL}$ $(8.6 \%$ at 20 years, $\mathrm{SE}=1.7 \%$ ) compared to patients with other NHL-entities $(3.4 \%$ at 20 years, $\mathrm{SE}=0.7 \%$, $P<0.0001)$, and in patients with known CPC $(19.6 \%$ at 20 years, $\mathrm{SE}=7.1)$ compared to patients without known CPC $(4.4 \%$ at 20 years, $\mathrm{SE}=0.7, P<0.0001)$ after exclusion of basal-cell carcinoma from the analysis.

\section{Multivariate analysis of risk factors}

In a stepwise Cox regression analysis taking into account risk factors significant in univariate analysis, which are listed in Table 1, only female sex (HR 1.9, 95\% CI: 1.2-2.9, $P=0.004$ ), CNS-involvement (HR 2.2, 95\% CI: $1.0-4.9, P=0.04)$, diagnosis of LBL (HR 2.6, 95\% CI: 1.7-4.0, $P<0.001$ ), and a known CPC (HR 11.2, 95\% CI: 5.5-22.8, $P<0.001)$ represented independent risk factors for the development of SMN (Table 2). Looking for the 

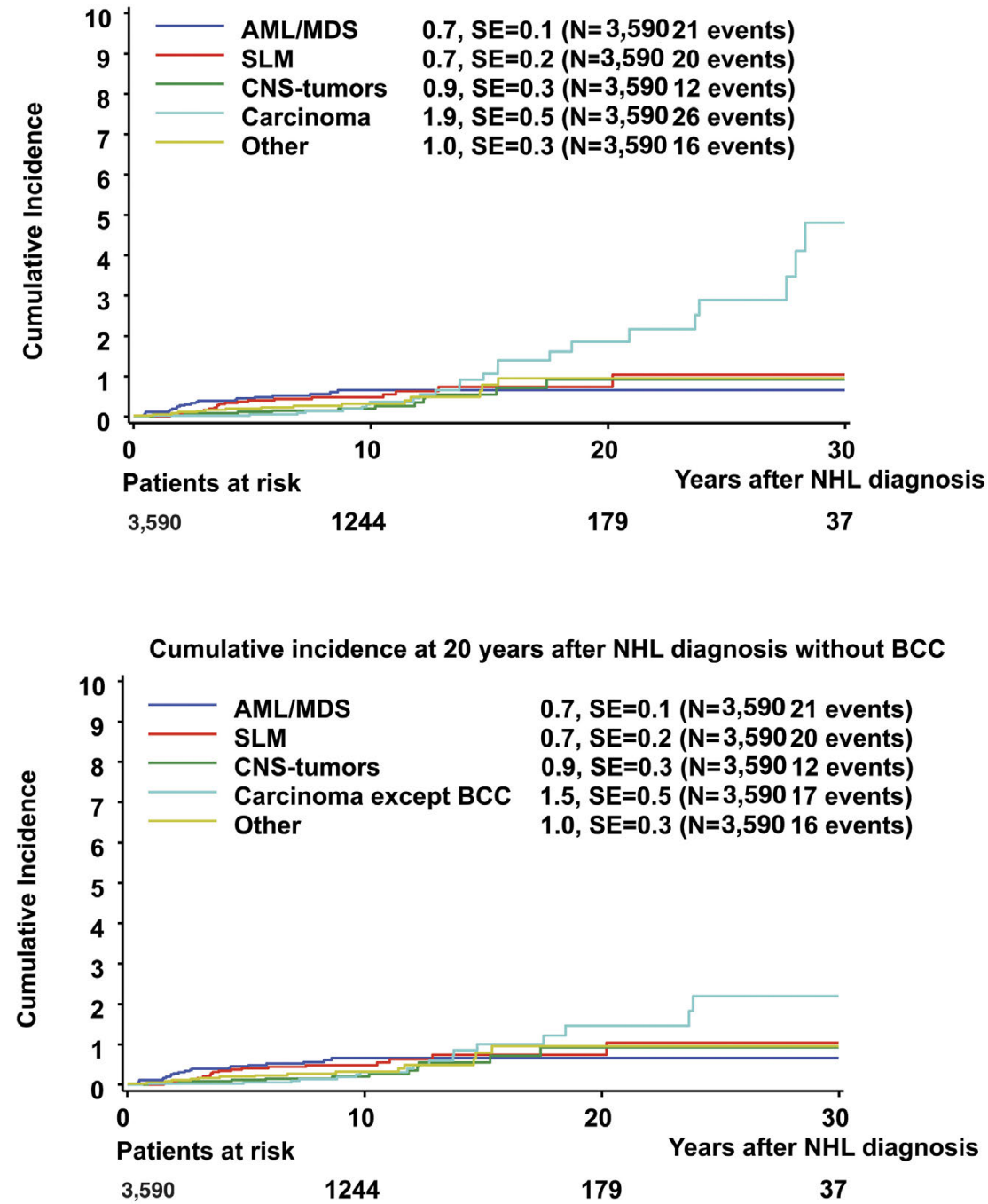

Figure 3. Estimated cumulative incidence of second malignant neoplasms (SML) 20 years after non-Hodgkin lymphoma (NHL)-diagnosis in $3,590 \mathrm{NHL}$-patients $<15$ years of age, treated in one of the consecutive NHL-BFM studies NHL-BFM-81, through EURO LB-02/B-NHL-04 according to the type of SMN. (A) Cumulative incidence of all SMN. (B) Cumulative incidence without basal-cell carcinoma (BCC). AML/MDS: acute myeloid leukemia/myelodysplastic syndrome; SE: standard error, SLM: second lymphoid malignancy.

risk factors for the development of AML/MDS, a diagnosis of $\mathrm{LBL}$ (HR 12.0, 95\% CI: 3.8-37.0, $P<0.001$ ), CNS-involvement (HR 5.2, 95\% CI: 1.5-18.5, $P=0.01$ ) and a known CPC (HR 8.8, 95\% CI: 1.2-67.3, $P=0.04)$ showed a significant association.

\section{Outcome after SMN}

Survival at 10 years after diagnosis of the SMN was $47 \%, \mathrm{SE}=6.4$. The worst prognosis carrying AML/MDS with a 10 -year survival of $26.5 \%, \mathrm{SE}=11.6$ was followed by CNS-malignancy with a 10 -year survival of $43.6 \%$, $\mathrm{SE}=15.5$. Patients with solid secondary tumors outside the CNS had a 10-year survival of $50.8 \%, \mathrm{SE}=13.0$ and patients with second lymphoid malignancy $57.8 \%$, $\mathrm{SE}=13.3$, respectively. Patients with known CPC had a survival of only $11.1 \%, \mathrm{SE}=10.5$. Nine patients (six with known CPC) with SMN developed a third malignant neoplasm (Tables 3 and 4).

\section{Discussion}

We presented the incidence and types of SMN as well as risk factors for the development of SMN in the to date largest cohort of patients who were treated for NHL as children according to subtype-specific BFM-type therapy regimens with stable cumulative drug doses over the analysis period. The estimated 20 -year cumulative incidence of $\mathrm{SMN}$ of $5.3 \pm 0.7 \%(4.8 \pm 0.7 \%$ without basal-cell carcinomas), among 3,590 patients is comparable to the one reported by Leung et al..$^{10}$ in a cohort of 497 children with NHL $(4.8 \pm 1.3 \%$ at 20 years). An analysis of 456 French and British 3-year survivors of pediatric NHL showed 25-year cumulative incidence of solid SMN of $10 \%$, and a relative risk of $12 \% .^{1}$ The CCSS group reported a 20 -year cumulative incidence of SMN of $3 \%$ (SIR of 3.9) in 1,082 5-year survivors of pediatric NHL. ${ }^{9}$ Since this study focused on solid-tumor SMN most of the AML/MDS that occured within 5 years of first NHL-diagnosis and all secondary lymphomas were not included which explains the somewhat lower cumulative incidence compared to our study. In an updated CCSS-report from 2010, a 30-year cumulative incidence of 5.8\% and SIR of $4.1 \%$ was reported for the same cohort of patients. ${ }^{8}$ The observation of an increasing cumulative incidence with longer observation time is reflected in our study by the missing plateau for secondary carcinomas after NHL-therapy.

Analyses in the US Surveillance, Epidemiology, and End Results (SEER) program showed a 5.3-fold elevated risk 
for SMN after NHL compared to the normal population in patients diagnosed before the age of 18 years with significantly higher risks for breast cancer and acute non-lymphoid leukemia in a cohort of 1,150 patients treated between 1973 and 2002.2 Another SEER analysis in a cohort of 1,832 5-year survivors of childhood NHL under 15 years of age at initial diagnosis reported SIR of 3.3 for SMN. ${ }^{4}$ Focusing on 4,310 NHL survivors treated with chemotherapy only, the CCSS-group reported a lower SIR of 2.4 for SMN occurring $\geq 5$ years from initial diagnosis without non-melanoma skin cancers. ${ }^{5}$ The evaluation of late outcomes in a cohort of 200 childhood NHL patients who had survived for $\geq 10$-years in the St Jude Lifetime Cohort Study revealed SIR of 6.3 for SMN, with none occurring in individuals not irradiated. ${ }^{12}$ We observed a higher SIR of 19.8 (without basal-cell carcinoma), which is similar to the SIR after acute lymphoblastic leukemia reported by the GCCR. ${ }^{6}$ Different inclusion criteria of patients regarding the age at initial diagnosis, varying restriction of the latency period to SMN-development, exclusion or inclusion of some SMN types (e.g., lymphoma), and possibly differences in the treatment regimen may influence variations in the SIR estimates. Younger age at initial diagnosis ( $<15$ years), no restriction of latency period to SMN-development, inclusion of patients with known CPC, and inclusion of second lymphoid malignancies explain a higher SIR in our cohort of patients treated by contemporary NHL-protocols with prophylactic brain irradiation only in LBL-patients. We cannot exclude that variations of the estimated cancer incidence in the control population might interfere regarding the different SIR-estimates as well.

Identified risk factors for SMN development in the published studies were female sex, ${ }^{9}$ previous radiotherapy, ${ }^{1,2,8,9,12}$ and use of epipodophyllotoxins (for development of sAML/MDS).,10 Cumulative incidence of SMN in our study was significantly higher in patients with LBL receiving ALL-type therapy compared to patients with other NHL-entities receiving B-NHL-type therapy, mainly attributable to the high incidence of sAML/MDS and carcinomas in LBL-patients. In contrast to Leung et al. ${ }^{10}$ and Inskip et al. ${ }^{2}$ we could not explain the occurrence of AML/MDS after LBL by excess use of epipodophyllotoxins, as these were not part of the BFM-ALL-type treatment regimen. The anthracycline doses of $240 \mathrm{mg} / \mathrm{m}^{2}$ doxorubicin-equivalent received by most LBL-patients could be potentially associated with the induction of AML/MDS. However, only four of 21 observed AML showed MLLrearrangements characteristic for topoisomerase-II inhibitors-induced AML. ${ }^{27,28}$ The majority of AML/MDS developed in patients with advanced stage T-LBL. An increased risk of AML was reported in pediatric patients after ALL with T-cell phenotype, too. ${ }^{29}$ Single reports on lineage switch in T-ALL relapsing as AML ${ }^{30}$ suggest a common origin of precursor $\mathrm{T}$ and myeloid cells. Our analysis of NHL-patients supports the above observations towards a possible common underlying mechanism predisposing some patients with T-cell precursor neoplasm to the development of AML/MDS.

In an analysis of late outcomes after treatment in 570 children with B-NHL ${ }^{11}$ who survived $\geq 5$ years from initial diagnosis, none of the 126 children treated with the contemporary "Lymphome Malin de Burkitt (LMB) therapy" between 1987 and 1999 without radiotherapy developed SMN. Patients treated with non-LMB protocols, including radiotherapy, had a SMN incidence rate of 2.2 per 1,000 person-years $\left(5,871\right.$ total person-years) ${ }^{11}{ }^{1}$ The inclusion of second lymphoid malignancies in our cohort which accounted for $37.8 \%$ of all SMN after B-NHL and the restriction of the reported SMN to $\geq 5$-year survivors in the earlier study ${ }^{11}$ can explain the higher risk estimate for patients after B-NHL type therapy in our analysis of $3.9 \%$.

The most frequent SMN after NHL in our as well as other analyses were solid tumors, the largest group consisting of carcinomas. Compared to the hematologic SMN, the cumulative incidence estimates for solid tumors do not show a plateau after 20 years which is in line with the observation of higher estimates in the CCSS studies with longer follow-up.

Several studies demonstrated a relationship between radiotherapy and solid SMN as exemplified by the occurrence of CNS-tumors after radiotherapy for a primary CNS-malignancy or after cranial radiotherapy for ALL. ${ }^{11,32}$ The latter was also observed in our study. In contrast to the findings of others, ${ }^{1,2,8,9,12}$ radiotherapy was not associated with development of SMN outside the CNS in our study. This can be explained by the omission of local radiotherapy early in the BFM-studies, and consecutively, very low numbers of patients exposed to radiotherapy outside the CNS in our cohort (38 of the 3,590 patients), precluding the detection of a significant impact on SMNdevelopment in the radiation field outside the CNS.

Several patterns noticed in our cohort suggest the possible existence of unknown familial cancer syndromes predisposing patients to NHL and solid neoplasia. ${ }^{33}$ Unlike previous reports ${ }^{1-10}$ we encountered an unusual high number of gastrointestinal tract-carcinomas, most of them occured after T-LBL. Interestingly, SIR of 67.3 for development of SMN of digestive tract after childhood NHL was also reported by Erhardt et al..$^{12}$ In three patients with gastrointestinal tract-carcinomas in our cohort a constitutional mismatch repair deficiency $(C M M R D)^{34}$ was diagnosed (Table 4). Nine patients developed $>2$ malignancies, in six of them a CPC was diagnosed, suggesting the presence of more, as yet undiscovered CPC. An European Inter-Group for Childhood Non-Hodgkin Lymphoma survey and iBFM-survey revealed a 10-year cumulative incidence of SMN of $24 \pm 5 \%$ among 151 children with CPC and NHL. ${ }^{35}$ An increased cancer risk (SIR 1.5) of siblings of patients with SMN after childhood cancer also support the theory of familial susceptibility for cancer development. ${ }^{3}$ Due to of the retrospective character of our study, a detailed family history of cancer burden can not be obtained or further genetic investigations cannot be easily performed. Further studies are needed to address this issue.

Skin cancer as SMN after childhood cancer has been mostly associated with radiotherapy ${ }^{36}$ Among the nine basal-cell carcinomas and four melanomas in our cohort, however, only six had received radiotherapy. Astonishingly, four basal-cell carcinomas developed after ALCL (without radiotherapy), an association which has not been observed so far.

Unexpectedly, in our analysis the third most frequent SMN after a childhood NHL was another lymphoid malignancy (Online Supplementary Table S3). A high incidence of second lymphoid malignancy after NHL has not been reported so far.

Most of the second lymphoid malignancies $(70 \%)$ occurred after mature B-NHL. Primary and secondary immune deficiencies, as well as defects of mismatch DNA- 
repair mechanisms bear susceptibility for development of NHL. ${ }^{37}$ In our study six patients with second lymphoid malignancy had a known CPC. For the remaining 14 patients either as yet unknown susceptibilities for development of lymphoma, or defects of immune surveillance might be responsible.

Following a large cohort of children with NHL enrolled in consecutive clinical studies with uniform treatment backbones is a strength of our study. The combined follow-up of patients by the NHL-BFM study group as well as the GCCR ensured a high probability to collect most of the SMN after NHL in childhood. Furthermore, all reports were medically validated. Careful analysis of second lymphoid tumors after NHL including comparison of tumor material from first and second tumors enabled us to distinguish second lymphoid malignancies from relapses. However, we cannot exclude an under-reporting of second lymphoid malignancies in cases of assumed relapses with similar histology and insufficient tumor material for molecular comparison. With a median follow-up time of 9.4 years (maximum 36 years) it is likely that we captured most of the hematologic SMN, but did not capture all second solid tumors. Despite the significant higher risk of SMN for patients with known CPC, it is also likely that we underestimated the frequency of CPC among children suffering from NHL, since CPC were not systematically screened for.

Genetic predisposition and immune suppression are important risk factors for the development of SMN in line with previously administered chemotherapy and/or radiotherapy. Therefore efforts should be made to identify individuals with CPC. The inclusion of basic immunological examinations and using standardized questionnaires ${ }^{38}$ about the personal and family history of the patients should be discussed for all children with NHL in order to provide a better basis for long-term patient care. Our study underscores the importance to continue to pay high attention on patient follow-up beyond the pediatric age. Patients and caregivers of survivors of childhood NHL should be aware of the specific risk factors for the development of SMN, and should be highly alert to early signs and symptoms of possible malignancy, such as carcinoma of the gastrointestinal tract or CNS tumor, in order to allow timely diagnosis. Also directed screening for SMN might be appropriate with the goal to improve the prognosis for the treatment of the second malignancy. In cases of late recurring lymphoid malignancies, the possibility of a SMN as opposed to relapse of the primary lymphoma should be considered with potential implications for the application of diagnostic tools (e.g., molecular genetics and clonality analysis) and treatment decisions.

In conclusion, our analysis revealed an increased risk for SMN after successful treatment of childhood NHL. The cumulative incidence of SMN was significantly higher in LBL-patients compared to the other subtypes of NHL. The diagnosis of LBL was an independent risk factor for the development of AML/MDS. Patients with known CPC had a significantly higher risk for the development of SMN than patients without known CPC. Solid malignancies were the most frequent SMN followed by AML/MDS and second lymphoid malignancy.

\section{Disclosures}

MS has received honoraria from $\mathrm{prIME}$ Oncology, is part of the speakers' bureau of Baxalta, has received research funding from Sigma Tau, Baxter and Medac; TK has a consulting and advisory role at Novartis; $A A$ has received honoraria from Jazz Pharmaceuticals and has a consulting and advisory role at Jazz Pharmaceuticals; $M Z, O M, U M, W K, I O, G M, F N, C S, U K$, $A R, B B$ and $W W$ have no conflicts of interest to declare.

\section{Contributions}

$A R, B B, W W$ and $O M$ designed the study; $A R, B B, F N, G M$, and WW were trial coordinators. IO, and WK did laboratory tests; $A A, A R, B B, F N, G M, M S, O M, T K, U K$, and $W W$ recruited patients for the BFM-trials coordinators; UM, MZ and CS established the dataset; $M Z$ performed statistical analysis; $A R, B B$, $M Z, W W$, and $O M$ analyzed data; $B B, W W$, and $O M$ wrote the paper; $O M$ had full access to all the data in the study and had final responsibility for the decision to submit for publication; all authors reviewed the draft of the paper submitted for publication.

\section{Acknowledgments}

We acknowledge the contribution of past and present coordinators and medical records technicians of the BFM study group for helping to establish this dataset. We thank all the clinicians who entered patients into the subsequent NHL-BFM trials and the children and families who agreed to take part.

\section{Funding}

This study was supported by the Forschungshilfe Station Peiper (Research Support Peiper), Germany, and the Deutsche Kinderkrebsstiftung (German Childhood Cancer Foundation) for the NHL-BFM Registry 2012 (DKS 2014.11 A/B), and the St. Anna Kinderkrebsforschung (Children's Cancer Research Institute), Austria.

\section{References}

1. de Vathaire F, Hawkins M, Campbell S, et al. Second malignant neoplasms after a first cancer in childhood: temporal pattern of risk according to type of treatment. $\mathrm{Br} \mathrm{J}$ Cancer. 1999;79(11-12):1884-1893.

2. Inskip P, Curtis RE. New malignancies following childhood cancer in the United States, 1973- 2002. Int J Cancer. 2007; 121(10):2233-2240.

3. Meadows AT, Friedman DL, Neglia JP, et al. Second neoplasms in survivors of childhood cancer: findings from the Childhood Cancer Survivor Study Cohort. J Clin Oncol. 2009;27(14):2356-2362.

4. Lee JS, DuBois SG, Coccia PF, Bleyer A,
Olin RL, Goldsby RE. Increased risk of second malignant neoplasms in adolescents and young adults with cancer. Cancer. 2016;122(1):116-123.

5. Turcotte LM, Liu O, Yasui Y, et al Chemotherapy and risk of subsequent malignant neoplasms in the childhood cancer survivor study cohort. J Clin Oncol. 2019;37(34):3310-3319.

6. Scholz-Kreisel P, Kaatsch P, Spix C, et al. Second malignancies following childhood treatment in Germany from 1980 to 2014 a registry-based analysis. Dtsch Arztebl Int. 2018;115(23):385-392.

7. Turcotte LM, Whitton JA, Friedman DL, et al. Risk of subsequent neoplasms during the fifth and sixth decades of life in the
Childhood Cancer Survivor Study Cohort. J Clin Oncol. 2015;33(31):3568-3575.

8. Friedman DL, Whitton J, Leisenring W, et al. Subsequent neoplasms in 5-year survivors of childhood cancer: the Childhood Cancer Survivor Study. J Natl Cancer Inst. 2010;102(14):1083-1095.

9. Bluhm EC, Roncers C, Hayashi RJ, et al. Cause specific mortality and second cancer incidence after non Hodgkin lymphoma: a report from the Childhood Cancer Survivor Study. Blood. 2008;111(8):4014-2021.

10. Leung W, Sandlund JT, Hudson MM, et al. Second Malignancy after treatment of childhood Non-Hodgkin Lymhoma. Cancer. 2001;92(7):1959-1966.

11. Ehrhardt MJ, Chen Y, Sandlund JT, et al 
O. Moser et al.

Late health outcomes after contemporary lymphome Main de Burkitt therapy for mature B-cell non-Hodgkin lymphoma: a report from the Childhood Cancer Survivor Study. J Chin Oncol. 2019;37(28):2556-2570.

12. Ehrhardt MJ, Sandlund JT, Zhang N, et al. Late outcomes of adult survivors of childhood non-Hodgkin lymphoma: a report from the St. Jude Lifetime Cohort Study. Pediatr Blood Cancer. 2017;64(6).

13. Sward JD, Wendland MM, Shrieve DC, Szabo A, and Gaffney DK. The risk of secondary malignancies over 30 years after the treatment of non-Hodgkin lymphoma. Cancer. 2006;107(1):108-115.

14. Pirani M, Marcheselli R, Marcheselli L, Bari A, Federico M, and Sochi S. Risk for secand malignancies in non-Hodgkin's lymphoma survivors: a meta-analysis. Ann Oncol. 2011;2(8)2:1845-1858.

15. Lennert K, Stein H, and Kaiserling E: Cytological and functional criteria for the classification of malignant lymphoma. Br J Cancer. 1975;31(Suppl 2):29-43.

16. Harris NL, Jaffe ES, Stein H, et al. A revised European-American classification of lymphoid neoplasms: a proposal from the International Lymphoma Study Group. Blood. 1994;84(5):1361-1392.

17. World Health Organization Classification of Tumours 2001. Pathology and genetics of tumours of haematopoietic and lymephoid tissues. IARC Press, Lyon.

18. Murphy SB. Classification, staging and end results of treatment in childhood non Hodgkin's lymphoma: dissimilarities from lymphomas in adults. Serin Oncol. 1980;7(3):332-339.

19. Woessmann W, Sideman K, Mann G, et al. The impact of the methotrexate administration schedule and dose in the treatmont of children and adolescents with Bcell neoplasms: a report of the BFM Group Study NHL-BFM95. Blood. 2005;105:948958.

20. Sideman K, Tiemann M, Schrappe M, et al. Short-pulse B-non-Hodgkin lymphomatype chemotherapy is efficacious treatment for pediatric anaplastic large cell lymphoma: a report of the Berlin-FrankfurtMunster Group Trial NHL-BFM 90. Blood. 2001;97(12):3699-3706.

21. Reiter A, Schappe M, Tiemann M, et al. Improved treatment results in childhood Bcell neoplasms with tailored intensification of therapy: a report of the Berlin-FrankfurtMunster Group Trial NHL-BFM 90. Blood. 1999;94(10):3294-3306.

22. Reiter A, Schappe M, Tiemann M, et al. Successful treatment strategy for Ki-1 anaplastic large-cell lymphoma of childhood: a prospective analysis of 62 patients enrolled in three consecutive BerlinFrankfurt-Munster group studies. J Chin Oncol. 1994;12(5):899-908.

23. Reiter A, Schrappe M, Ludwig WD, et al. Favorable outcome of B-cell acute lymphoblastic leukemia in childhood: a report of three consecutive studies of the BFM group. Blood. 1992;80(10):2471-2478.

24. Kalbfleisch J, Prentice RL. The statistical analysis of failure time data. New York, NY: John Willy. 1980;163.

25. Kaplan EL and Meier P. Non-parametric estimation from incomplete observations. J Am Stat Assoc. 1958;53(282):457-481.

26. van Dongen JJ, Langerak AW, Bruggemann $M$, et al. Design and standardization of PCR primers and protocols for detection of clonal immunoglobulin and T-cell receptor gene recombination in suspect lymphoproliferations: report of the BIOMED-2 Concerted Action BMH4-CT98-3936. Leukemia. 2003;17(12):2257-2317.

27. Leone G, Voso MT, Sick S, Morosetti, and Pagano L. Therapy related leukemias: susceptibility, prevention and treatment. Leek Lymphoma. 2001;41(3-4):255-276.

28. Puri CH, Relling MV. Topoisomerase II inhibitor-related acute myeloid leukaemia. Br J Haematol. 2000;109(1):13-23.

29. Löning L, Zimmerman M, Reiter A, et al. Secondary neoplasms subsequent to BerlinFrankfurt-Münster therapy of acute lymphoblastic leukemia in childhood: signifiscantly lower risk without cranial radiother- apy. Blood. 2000;95(9):2770-2775.

30. Paganin M, Buldini B, Germano G, et al. A Case of T-cell Acute Lymphoblastic Leukemia Relapsed As Myeloid Acute Leukemia. Pediatr Blood Cancer. 2016;63(9):1660-1663.

31. Macle M, Scelo G, Pastor G, et al. Risk of second malignant neoplasms after childhood leukemia and lymphoma: an internetional study. J Natl Cancer Inst. 2007;99(10):790-800.

32. Relling MV, Rubnitz JE, Rivera GK, et al. High incidence of secondary brain tumours after radiotherapy and antimetabolites. Lancet. 1999;354(9172):34-39.

33. Ripperger T, Schlegelberger B. Acute lymphoblastic leukemia and lymphoma in the context of constitutional mismatch repair deficiency syndrome. Eur J Med Genet. 2016;59(3):133-142.

34. Wimmer K, Kratz CP, Vase HF, et al. EUConsortium Care for CMMRD (C4CMMRD). Diagnostic criteria for constitutional mismatch repair deficiency syndrome: suggestions of the European consortium 'care for CMMRD' (C4CMMRD). J Med Genet. 2014;51(6):355-365.

35. Attarbaschi A, Carrara E, Ala O, et al. Non-Hodgkin lymphoma and pre-existing conditions: spectrum, clinical characterstics and outcome in 213 children and adolescents. Haematological. 2016; 101(12):1581-1591.

36. Whiles L, Black J, Alborg AJ. Nonmelanoma skin cancer and the risk of second primary cancers: a systematic review. Cancer Epidemiol Biomarkers Prev. 2010;19(7):1686-1695.

37. Levine AM. Lymphoma complicating immunodeficiency disorders. Ann Oncol. 1994;5(Suppl 2):S29-35

38. Ripperger T, Bielack SS, Borkhardt A, et al. Childhood cancer predisposition syndromes-A concise review and recommendations by the Cancer Predisposition Working Group of the Society for Pediatric Oncology and Hematology. Am J Med Genet. 2017;173(4):1017-1037.

1400

haematological | 2021; 106(5) 